\title{
Modern fracture mechanics for structural optimization with incomplete information
}

\author{
N.V. Banichuk ${ }^{\mathrm{a}}$, S.Yu. Ivanova and E.V. Makeev \\ Institute for Problems in Mechanics, Russian Academy of Scienses, Moscow, Russia
}

Received 30 October 2007, Accepted 15 February 2008

\begin{abstract}
Questions described in this paper are concerned with the shape optimization of brittle or quasi-brittle (axisymmetric) elastic shells. These questions take into consideration the possibilities of crack arising and damage accumulation in the process of application of cyclic load to the shell structure. Initial structural defects, arising cracks and damage accumulation are characterized by incomplete information concerning initial crack sizes, crack position and its orientation. In this context we develop the statements of the optimization problems based on guaranteed approach for the considered problems with incomplete information. For many realistic cases it is reasonable to use variants of the mini-max optimization, named as optimization for "the worst case scenario". Considered in this paper the structural optimization problems consist in finding of the shape and thickness distribution of axisymmetric quasi-brittle elastic shells with arising cracks in such a way that the cost functional (volume or weight of the shell material) reaches the minimum, while satisfying some constraints on the stress intensity factor and geometrical constraints. In the case of cycling loadings, we consider the number of loading cycles before fracture as the main constraint.
\end{abstract}

Key words: Shells; cracks; structural design; shape optimization; incomplete information.

\section{Introduction}

In the theory of optimal structural design there are several principal directions. One of these directions is devoted to conventional problems of structural optimization under strength constraints without taking into account initial damages and the possibilities of cracks arising and their growth. However, because the elements of many important structures are usually brittle or quasi-brittle, they are prone to cracking under very low applied stresses. Cracking not only reduces structural stiffness, but can lead to other undesirable effects such as delamination and global fracture. A few studies have been devoted to this class of problems of brittle and quasi-brittle bodies optimization on the basis of modern fracture mechanics criterion. Thomsen et al. [1], Thomsen and Karihaloo [2], Wang and Karihaloo [3] investigated some problems of crack appearance in the considered bodies. Crack appearance was also taken into account by Papila and Haftka [4] and Vitali et al. [5]. In these investigations all parameters of crack appearing have been supposed as given. The problem of the shape design of complex cracked shells under the optimization criteria of the weight of the structure and the strength constraint on a critical stress intensity factor has been studied by Abdi et al. [6]. In this paper the optimal cracked structure is that with minimum weight and with a stress intensity factor which does not reach the critical stress intensity factor.

\footnotetext{
${ }^{a}$ Corresponding author: banichuk@ipmnet.ru
}

In the most cases the number of cracks, their position, orientation, size and modes (opening cracks, shear cracks, mixed cracks) are unknown beforehand and so the optimal structural design (especially for brittle and quasibrittle bodies) intrinsically contains uncertainty factors or randomness. In these cases it is difficult or impossible in principle to obtain complete information concerning the problem parameters and to formulate the structural optimization problem as the classical pure deterministic problem. In such cases to apply fracture mechanics representation in the design process and to formulate optimal design problem we can use min-max guaranteed approaches, probabilistic approaches and some mixed probabilistic-guaranteed approaches, developed early in the theory of optimal control and in the theory of differential games.

Banichuk [7,8] formulated general structural optimization problem under fracture mechanics constraints adopting arbitrary crack-appearance using min-max guaranteed approach. Some probabilistic approaches for optimal design of structures and structural elements and mixed probabilistic-guaranteed approaches have been developed by $\mathrm{Yu}$ et al. [9], Banichuk et al. [10,11]. In what follows [12] investigated macrofailure criterion and performed the optimization of homogeneous structures and composite structures with edge delamination.

An important damage scenario is realized when a through - the thickness cracks are propagated under cyclic loading. Corresponding questions have been 
investigated in the context of optimal design of beams and beam-like structures by Banichuk et al. [13] and Serra [14]. In this context we can mention also the paper by Lyubimov and Makarenko [15] devoted to the optimization problem of a reinforced plate with a crack.

\section{Basic relations and the problem of the optimal structural design}

In the paper we consider the elastic shell that has the form of surface of revolution. The position of the meridian plane is defined by the angle $\theta$, measured from datum meridian plane and the position of a parallel circle is defined by the angle $\varphi$ made by the normal to the surface and the axis of rotation (see Fig. 1) or by the coordinate $x$, measured along the axis of rotation, $0 \leqslant x \leqslant L, L$ - given value. The meridian plane and the plane perpendicular to the meridian are the planes of principal curvatures. The geometry of the shell is defined when a shape of the middle surface is given. We restrict our consideration to an axially symmetric shape of a middle surface (a profile of each cross-section of a shell is a circle) and will use a distance $r(x)$ from the axis of rotation to a point of the middle surface as the variable describing the shape of the middle surface. These variables $r(x)$ and the thickness of the shell $h(x)$ will be considered as the design variables. The geometrical relations between meridian radius of curvature $r_{\varphi}(x)$, circumferential radius of curvature $r_{\theta}(x)$ and the radius $r(x)$ are given by the following expressions

$$
\begin{gathered}
r_{\varphi}=-\left(1+\left(\frac{d r}{d x}\right)^{2}\right)^{3 / 2}\left(\frac{d^{2} r}{d x^{2}}\right)^{-1} \\
r_{\theta}=r \sqrt{1+\left(\frac{d r}{d x}\right)^{2}} \\
r=r_{\theta} \sin \varphi, \frac{d r}{d \varphi}=r_{\varphi} \cos \varphi, \frac{d x}{d \varphi}=r_{\varphi} \sin \varphi
\end{gathered}
$$

It is assumed that a through crack can be arisen in the shell during its manufacturing or exploitation and it is taken into account that the material of the shell is quasibrittle. The arisen crack is supposed to be rectilinear and its length is very small with respect to characterized geometric sizes of the shell without the restriction on the location of the crack in the shell, its orientation and its initial length $l_{i} \leqslant l_{c r}$. The value $l_{c r}$ determines the moment when the global fracture can be realized.

The parameters of the crack characterizing its size, location, shape and orientation (in the case of crack appearance) can not be specified rigorously. Taking this into account we specify only the set $\Lambda$ which contains all possible vectors

$$
\omega=\left\{l, x_{c}, \alpha\right\}
$$

where $x_{c}$ - coordinate of the crack center, $l$ - length of the crack, $\alpha$ - angle setting the crack inclination with respect to the meridian. The second coordinate $\theta_{c}$ of the crack

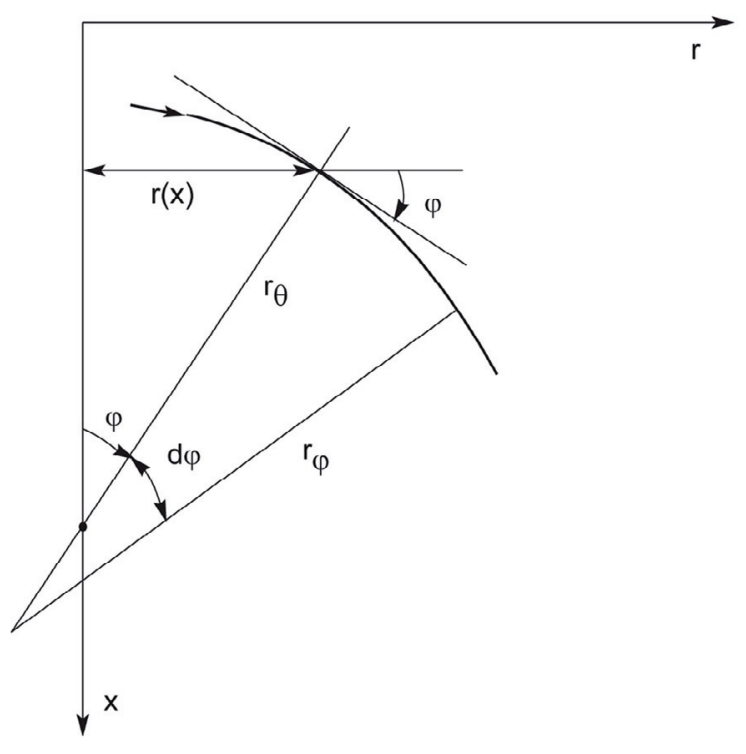

Fig. 1. Geometric terminology of the axisymmetric shell.

midpoint is nonessential and omitted because we consider axisymmetric problems and admit all possible locations of the crack in parallel direction $\left(0 \leqslant \theta_{c} \leqslant 2 \pi\right)$. If $\alpha=0$, the crack is oriented in the meridian direction (axial crack) and if $\alpha=\pi / 2$, the crack peripherally oriented in parallel direction. We shall use the following condition

$$
\omega \in \Lambda \text {. }
$$

Accepted assumptions and available additional data concerning the most dangerous parts of the shell suggest that the set $\Lambda$ to be considered as given.

The safety condition as it is well known from quasibrittle fracture mechanics $[16,17]$, is written as

$$
K_{1} \leqslant K_{1 C}
$$

and is approximated by the modified inequality constraint $[18,19]$

$$
K_{1} \leqslant K_{1 \varepsilon}, K_{1 \varepsilon}=K_{1 C}-\varepsilon, \varepsilon \geqslant 0
$$

where $K_{1}$ is the stress intensity factor occurring in asymptotic representations of the stresses nearby the crack tip (in the opening mode) and $K_{1 C}-$ given quasi-brittle strength constant (toughness of the material). Here we introduced small positive parameter $\varepsilon$ and reduced meaning $K_{1 \varepsilon}$ of the limiting value of the stress intensity factor $K_{1}$. This approximation is used for convenience of application of the shape optimization procedure.

After application of cyclic loading the initial crack goes ahead, and its length $l$ monotonically increases $\left(l_{i} \leqslant l \leqslant l_{c r}\right)$. The process of the fatigue crack growth 
under cyclic loading can be adequately characterized in the following form $[16,17]$

$$
\begin{gathered}
\frac{d l}{d n}=C\left(\Delta K_{1}\right)^{m} \\
l_{i} \leqslant l \leqslant l_{c r}, \quad 0 \leqslant n \leqslant n_{c r} .
\end{gathered}
$$

Here $C$ and $m(2 \leqslant m \leqslant 4)$ are some material constants, $K_{1}$ - is the stress intensity factor for opening cracks and the increment $\Delta K_{1}$ is given by

$\Delta K_{1}=\left(K_{1}\right)_{\max }-\left(K_{1}\right)_{\min }=\left(K_{1}\right)_{p=p_{\max }}-\left(K_{1}\right)_{p=p_{\min }}$.

Here $\left(K_{1}\right)_{\max }$ and $\left(K_{1}\right)_{\min }$ are the maximum and minimum values of the stress intensity factor $K_{1}$ in any given cycle, respectively.

The ordinary differential equation (7) defines the quasistatical process of crack growth and determines the dependence of the crack length $l$ on the number of cycles $n$. This equation is valid up to the moment, when

$$
n=n_{c r}, \quad l=l_{c r}
$$

and the unstable crack growth (catastrophic fracture of the shell) is attained. To find $l_{c r}$ we will use the fracture criterion

$$
\left(K_{1}\right)_{l=l_{c r}, p=p_{\max }}=K_{1 \varepsilon} .
$$

In what follows we will assume that not only the initial crack but also all temporary cracks $\left(l_{i} \leqslant l \leqslant l_{c r}\right)$ are rectilinear and that the crack length $l$ is bigger than $h$ and is small with respect characterized size $r_{m}$ of the shell.

In the considered design process the longevity constraint can be taken as $n_{c r} \geqslant n_{*}$, where $n_{*}$ is a given minimum value of the number of cycles. Taking into account incompleteness of the information concerning the possible location of initial cracks we can rewrite the longevity constraint in the following manner

$$
\min _{\omega \in \Lambda} n_{c r} \geqslant n_{*}
$$

Considered optimization problem consists of finding the shape $r=r(x)$ and the thickness distribution $h(x)$, $(0 \leqslant x \leqslant L)$ of the shell such that the volume of the shell material

$$
J=2 \pi \int_{0}^{L} r h\left(1+\left(\frac{d r}{d x}\right)^{2}\right)^{1 / 2} d x
$$

is minimized, while satisfying the longevity constraint (12) and additional geometrical constraint

$$
r(x) \geqslant r_{g}(x), \quad 0 \leqslant x \leqslant l
$$

where $r_{g}(x) \geqslant 0$ - given function. This restriction do not change the common sense of the problem but permits to avoid possible singularities.

The shape of the shell will be called optimal if for any shell with a smaller weight it is possible to select a vector of unknown parameters $\omega$ belonging to the admissible set $\Lambda$ such that some assigned constraints have been violated.

Taking into account that the longevity constraint (12) is reduced to the system of inequalities for stress components $[20,21]$ we shall consider (non-dimensional) problem of minimization of the functional (13) under the following constraints

$\psi_{1}=\frac{\gamma}{h r} \sqrt{1+\alpha\left(\frac{d r}{d x}\right)^{2}} \int_{x}^{1} r h \sqrt{1+\alpha\left(\frac{d r}{d x}\right)^{2}} d x-\sigma_{1} \leqslant 0$

$$
\begin{aligned}
\psi_{2}=\frac{\beta}{h}\left(r h \frac{d r}{d x}\right. & -\frac{\frac{d^{2} r}{d x^{2}}}{\sqrt{1+\alpha\left(\frac{d r}{d x}\right)^{2}}} \\
& \left.\times \int_{x}^{1} r h \sqrt{1+\alpha\left(\frac{d r}{d x}\right)^{2}}\right)-\sigma_{2} \leqslant 0
\end{aligned}
$$

$$
\begin{gathered}
\psi_{3}=-\frac{\beta}{h}\left(r h \frac{d r}{d x}-\frac{\frac{d^{2} r}{d x^{2}}}{\sqrt{1+\alpha\left(\frac{d r}{d x}\right)^{2}}}\right. \\
\left.\times \int_{x}^{1} r h \sqrt{1+\alpha\left(\frac{d r}{d x}\right)^{2}}\right)-\sigma_{1} \leqslant 0 \\
r_{g} \leqslant r(x) \leqslant 1, \quad 0 \leqslant x \leqslant 1 \\
h_{\min } \leqslant h(x) \leqslant 1, \quad 0 \leqslant x \leqslant 1 \\
\psi_{4}=V_{0}-\pi \int_{0}^{1} r^{2} d x \leqslant 0 .
\end{gathered}
$$

Here $\alpha, \beta, \gamma, \sigma_{1}, \sigma_{2}, V_{0}, h_{\text {min }}$ are given dimensionless parameters of the problem.

Note that the problem of brittle and quasi-brittle shape optimization formulated in this paragraph is investigated for the class of axisymmetric shells that is important as from theoretical as from practical points of view. Besides this class of optimization problems is characterised by the possibility to arrive to one-dimensional formulation of the optimization problem.

\section{Computations of the optimal shapes by genetic algorithm}

To find the solution of the optimization problem (13), (15)-(20) for the axisymmetric shell under the action of force of gravity and the reaction force we shall use the method of penalty functions in combination with genetic algorithm. To this purpose we introduce the augmented functional

$$
J^{a}=J+\sum_{i=1}^{4} \mu_{i} J_{i}
$$




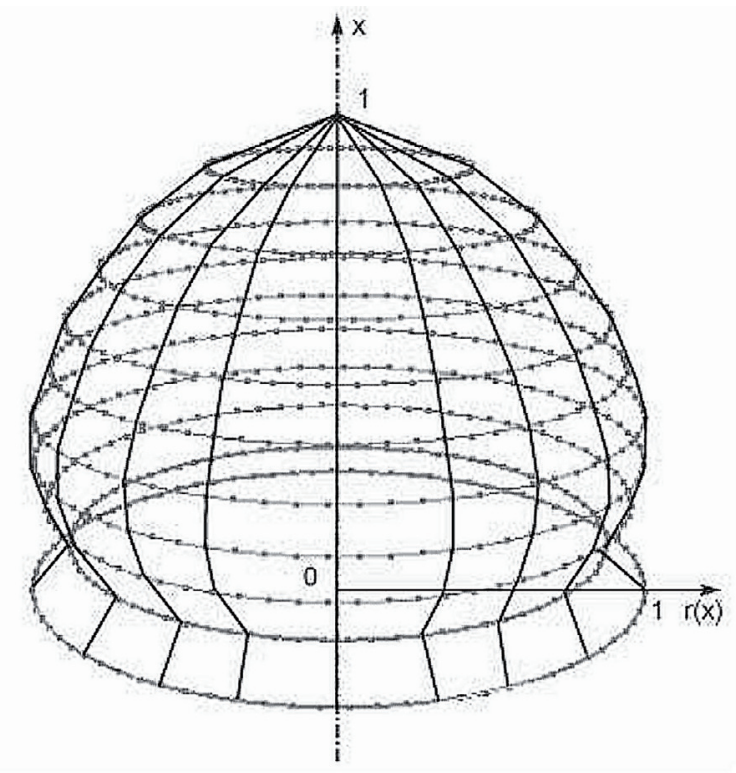

Fig. 2. A typical optimal shape of the axisymmetric shell.

where $\mu_{i}$ - arbitrary positive constants and $J_{i}$ - penalty functionals defined as

$$
\begin{gathered}
J_{i}=\int_{0}^{1} \Psi_{i} d x i, i=1,2,3 \quad \Psi_{i}=\left\{\begin{array}{l}
0, \text { if } \psi_{i} \leqslant 0 \\
\psi_{i}, \text { if } \psi_{i}>0
\end{array}\right. \\
J_{4}=\left\{\begin{array}{l}
0, \text { if } \psi_{4} \leqslant 0 \\
\psi_{4}, \text { if } \psi_{4}>0 .
\end{array}\right.
\end{gathered}
$$

Thus the original problem of optimal design is reduced to finding of design variables $r(x), h(x)$ satisfying inequalities (18), (19) and minimizing the augmented functional (20).

Optimal shape and thickness distributions of axisymmetric shells have been found numerically (one of the optimal projects is shown in Fig. 2) with application of the genetic algorithm [22].

It is supposed that the interval $[0,1]$ of the variable $x$ is divided by the points $x_{i}, i=1,2, \ldots, n$ into $n-1$ subintervals of equal length $\Delta x$. Populations under consideration consist of $N$ individuals. Each $j$-individual of the population is described by two sets of values $r(j, i), h(j, i)$ representing the meanings of design variables at the nodes. The "best" individual, i.e. the sets $r(j, i), h(j, i)$ minimizing augmented functional, is sought with application of genetic algorithm. The first step of the algorithm consists in initialization of population that is performed by means of assignment of random values taken from $\left[r_{g}(i), 1\right]$ to each element $r(j, i)$ and from $\left[h_{m i n}, 1\right]$ to each element $h(j, i)$. The values $r(j, i)$ at the nodes with numbers $i=1$ amd $i=n$ are fixed. For created individuals $(j=1, \ldots, N)$ of initial population we complete augmented functional $J^{a}(j)$ and find the individual having the minimal value of the functional. Using initial data on the next steps of the algorithm it is possible to determine new population consisting of $N$ individuals and to perform in what follows successive minimization of the functional $J^{a}$. At the second step of the algorithm we perform successive selection of $N / 2$ individual pairs - "parents" to obtain $N / 2$ pairs of individuals - "children", which constitute new population. Selection of the first parent ("a") is performed by the following manner. Some natural number $N^{T}$ is choused and then $N^{T}$ individuals are selected by the random way. From this set of individuals we preserve and use only one individual having the minimal value of augmented functional $J^{a}$. Similarly we find the second parent ("b") and put together the first pair of individuals. All together we compose $N / 2$ of such pairs. The third step of the algorithm consists in obtaining of two children from each parent pair of individuals. To this purpose we take some constant value from the interval $[0,1]$, that is called as crossover probability $p_{c o}$. Then for each parent pair it is generated random number $p_{r}$ from interval $[0,1]$ and random natural number $m_{r}$ from $[1, \ldots, n]$. If it is appeared that $p_{r} \leqslant p_{c o}$ then the values of design variables of children at the nodes $i=1,2, \ldots, m_{r}$ are copied from their parents "a" and "b", but the meanings of these values at the nodes $i=m_{r}+1, \ldots, n$ are obtained with the help of crossover. The latter means that for child "a" we copy the values in the corresponding nodes of the parent "b" and vice versa. Successive sorting of all parent pairs and performing of described operations lead to obtaining of $N$ individuals - children, that compose new population. The fourth step of the algorithm consists in mutation of the obtained new population. This step is necessary not to stick at the local minimum of the considered functional. To realize the mutation procedure we take some small enough $(\sim 0.005)$ parameter $p_{m}$ (probability of mutation). Then for all nodes of each individual of the population we generate the random number $p_{r}$ from the interval $[0,1]$. If $p_{r} \leqslant p_{m}$ then the value of design variable at this node is replaced by the arbitrary value, satisfying given constraints. Mutation procedure is not performed for the values $r(j, i)$ at the nodes with numbers $i=1$ amd $i=n$. For obtained new population we compute the functionals $J^{a}(j)$ and select the best individual. Then we go to the second step of applied algorithm. Note, that if the best child from new population is appeared to be worse then the best parent from previous population then we replace it by this parent. Thus we arrive the monotony in the process of finding of global minimum. Finish of the algorithm work is when the number of populations is large enough for convergence of the functional to be minimized.

With application of described algorithm we determined the optimal shapes of axisymmetric shell for the following values of problem parameters: $n=11, N=10$, $N^{T}=4, p_{C O}=0.5, p_{m}=0.05 \quad \alpha=1, \beta=0.1$, $\gamma=0.1, h_{\text {min }}=0.1, r_{g}(x)=0, \sigma_{1}=\sigma_{2}=1, \mu_{i}=1$ $(i=1, \ldots, 4)$. Calculations were completed after generations of 10000 populations. Optimal shapes of shells for boundary conditions $r(0)=1, r(1)=0$ and given volumes $V_{0}=2, V_{0}=1.5, V_{0}=1$ are represented by the curves 1, 2, 3 in Figure 3. Dependence of the functional $J^{a}$ on the number of population of genetic algorithm (number of iteration) characterizing the speed of convergence 
$1 \mathrm{x}$

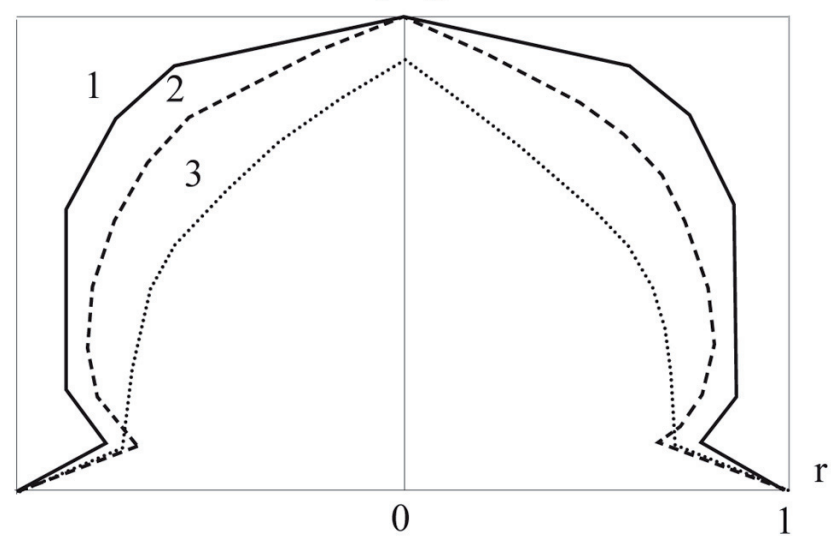

Fig. 3. Optimal shapes distributions for different values of volume of the shell.

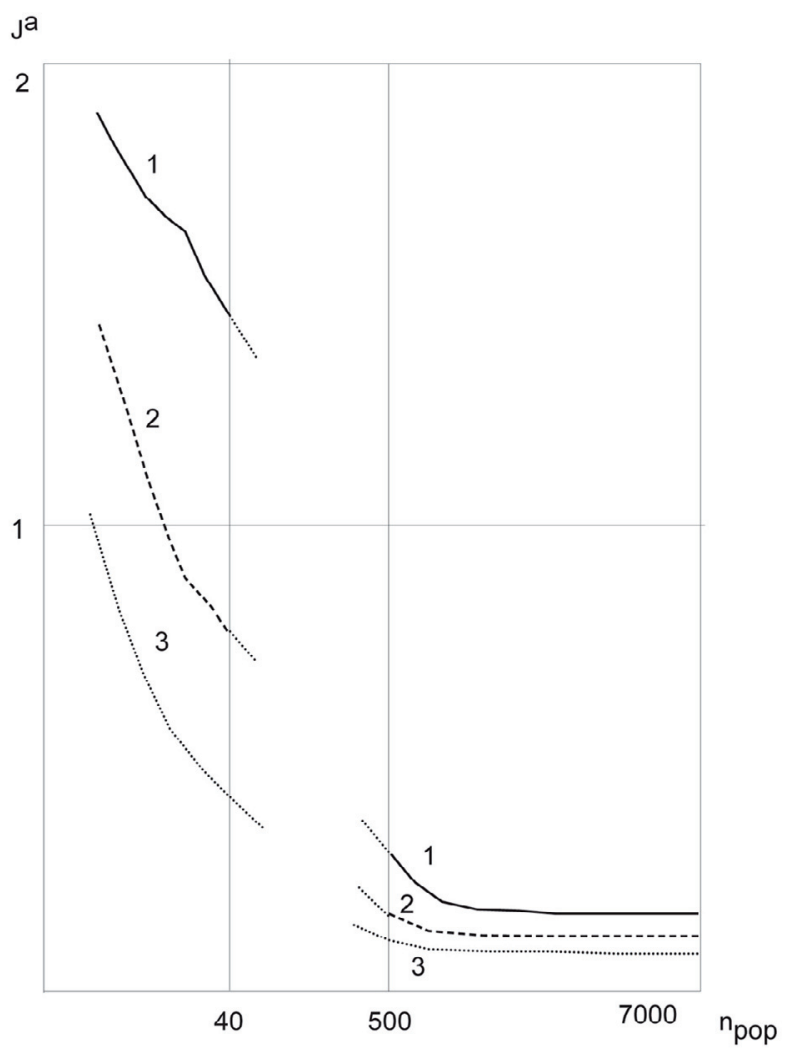

Fig. 4. Dependence of the functional $J^{a}$ on the number of population of genetic algorithm.

is shown in Figure 4. Optimal shapes of shells for $V_{0}=2$ are shown in Figure 5 by curves 1 (for $r(0)=1$ ) and 2 (for $r(0)=0.9$ ). In all considered cases the optimal distributions of shell thickness have been also found. In Figure 6 the curves 1 and 2 correspond to optimal thickness distributions for $r(0)=1, r(1)=0$ and two values of shell volume $V_{0}=2$ and $V_{0}=1.5$, respectively. We can see the increasing of thickness along the boundary circle

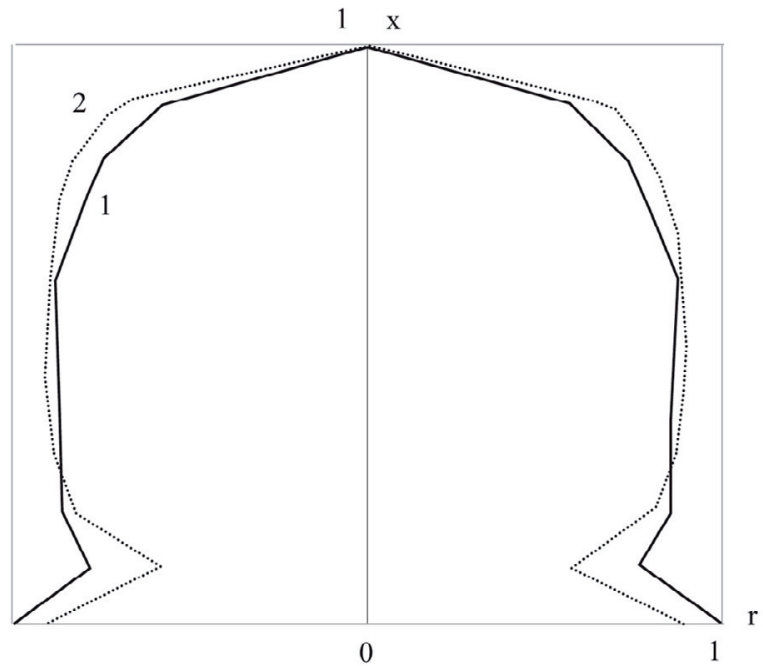

Fig. 5. Optimal shapes distributions for $V_{0}=2$ and different values of $r(0)$.

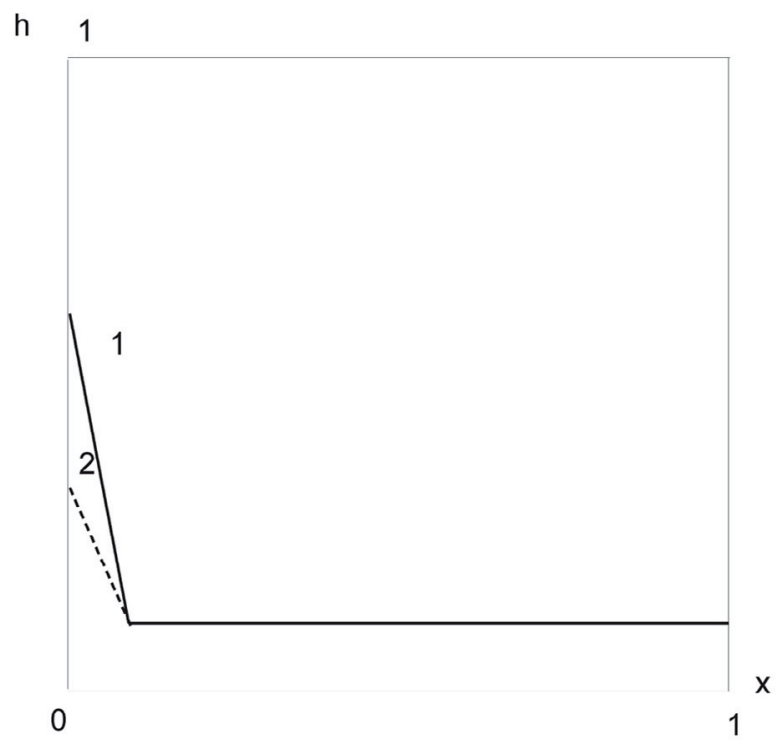

Fig. 6. Optimal thickness distributions for $V_{0}=2$ and $V_{0}=1.5$.

with radius $r_{0}=r(0)$. This is the characteristic feature of all considered optimal projects.

\section{Conclusions}

In this paper the problem of optimal shell design based on fracture mechanics of brittle and quasi-brittle bodies was considered. Some possibilities of effective application of minimax (guaranteed) approach and "worst case scenario" were demonstrated. The original optimization problem with uncertainties in damage characteristics (crack position and orientation) was reformulated using the transformation of the longevity constraint into the 
direct constraints imposes on the stresses and was effectively solved with the help of application of the penalty function method and genetic algorithm. Performed investigations are related to designing of axisymmetric shells loaded by axisymmetric forces. However, the optimization method described in this paper can also be applied to problems of optimal design of shells under more general geometrical and loading conditions in the frame of guaranteed approach.

\section{Acknowledgements}

The paper is performed under support of RFBR (grants № 05-08-18094-a, № 07-08-08010-3, № 08-08-00025-a), RAS Program № 14 ("Accumulation of damages, fracture and ..."), Program of Support of Leading Scientific Schools (grant № 169.2008.1).

\section{References}

1. N.B. Thomsen, J. Wang, B.L. Karihaloo, Structural Optimization 8, 9 (1994)

2. N.B. Thomsen, B.L. Karihaloo, J. Am. Ceram. Soc. 78, 3 (1995)

3. J. Wang, B.L. Karihaloo, Composite Structures 32, 453 (1995)

4. M. Papila, R.T. Haftka, Structural Optimization 25, 327 (2003)

5. R. Vitali, R.T. Haftka, B.V. Sankar, Struct. Multidisc. Optim. 23, 347 (2002)

6. R. Abdi, M. Touratier, P. Convert, Optimal design for minimum weight in cracked pressure vessel of a turboshaft, in: Communications in Numerical Methods in Engineering (1996), pp. 271-280
7. N.V. Banichuk, Analete Stiintifice, Universitatii. OVIDIUS, Constanta 5, 13 (1996)

8. N.V. Banichuk, Mechanics of Structures and Machines 26, $365(1998)$

9. X. Yu, K.K. Choi, K.X. Chang, Structural Optimization 14, 81 (1997)

10. N.V. Banichuk, F. Ragnedda, M. Serra, Meccanica 34, 29 (1999)

11. N.V. Banichuk, F. Ragnedda, M. Serra, Mechanics Based Design of Structures and Machines 31, 459 (2003)

12. A. Borovkov, V. Palmov, N. Banichuk, E. Stein, V. Saurin, F. Barthold, Yu. Misnik, Int. J. Comput. Civil Struct. Eng. 1, 91 (2000)

13. N.V. Banichuk, F.J. Barthold, M. Serra, Meccanica 40, 135 (2005)

14. M. Serra, Struct. Multidisc. Optim. 19, 159 (2000)

15. A.K. Lyubimov, F.V. Makarenko, Probability approach to the optimization problem of a reinforced plate with a crack, in Applied Problems of Strength and Plasticity (KMK Scientific Press, Moscow, 1996), pp. 120-131

16. K. Hellan, Introduction to Fracture Mechanics (New York, Mc-Graw-Hill, 1984)

17. M.F. Kanninen, C.H. Popelar, Advanced Fracture Mechanics (Oxford University Press, New York, 1985)

18. N.S. Streletskii, Izv. AN SSSR, OTN 1 (1947)

19. V.V. Bolotin, Statistical Methods in Structural Mechanics (Holden-Day, inc., San-Francisco, 1969)

20. N.V. Banichuk, S.Yu. Ivanova, E.V. Makeev, A.V. Sinitsin, Mechanics Based Design of Structures and Machines 33, 253 (2005)

21. N.V. Banichuk, P. Neittaanmäki, Mechanics Based Design of Structures and Machines 35, 76 (2007)

22. D.E. Goldberg, Genetic Algorithm in Search, Optimization and Machine Learning (Westley Publ. Comp., inc., M.A. 1989), p. 154 